\title{
Réécritures posthumes de Tchicaya U Tam'si entre Brazzaville et Pointe-Noire
}

Pierre Leroux

\section{OpenEdition}

\section{Journals}

Electronic version

URL: http://journals.openedition.org/coma/518

DOI: 10.4000/coma.518

ISSN: 2275-1742

\section{Publisher}

Institut des textes \& manuscrits modernes (ITEM)

\section{Electronic reference}

Pierre Leroux, "Réécritures posthumes de Tchicaya U Tam'si entre Brazzaville et Pointe-Noire", Continents manuscrits [Online], 4 | 2015, Online since 15 March 2015, connection on 16 April 2021. URL: http://journals.openedition.org/coma/518; DOI: https://doi.org/10.4000/coma.518

This text was automatically generated on 16 April 2021.

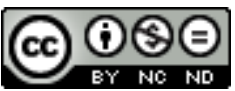

Continents manuscrits - Génétique des textes littéraires - Afrique, Caraîbe, dispora est mis à disposition selon les termes de la licence Creative Commons Attribution - Pas d'Utilisation

Commerciale - Pas de Modification 4.0 International. 


\title{
Réécritures posthumes de Tchicaya U Tam'si entre Brazzaville et Pointe- Noire
}

\author{
Pierre Leroux
}

1 En août 2012, à Brazzaville, vingt-huit bustes à l'effigie de personnalités congolaises sont dévoilés au public. Parmi ceux-ci, des figures historiques comme la prophétesse Béatrice Kimpa Vita, des hommes politiques comme Patrice Lumumba, mais aussi des écrivains et des artistes. Le poète Tchicaya U Tam'si est ainsi représenté aux côtés entre autres - de ses compatriotes Sylvain Bemba et Sony Labou Tansi ${ }^{1}$. Cet hommage des autorités à un enfant du pays témoigne d'une reconnaissance paradoxale, car, si nombreux sont ceux qui savent qu'il a été poète, les véritables lecteurs de son œuvre sont rares. C'est justement d'un questionnement sur la place occupée par le poète, romancier et dramaturge dans le paysage littéraire de son pays d'origine qu'est née la réflexion présentée dans cet article ${ }^{2}$.

2 Une part importante des commentateurs de l'œuvre de Tchicaya inscrit sa démarche critique dans une entreprise de réhabilitation, voire de réparation, si bien que l'analyse des textes prend parfois l'allure d'un devoir de mémoire envers un aîné injustement négligé. Sans parler du volume d'hommages coordonné par Nino Chiapano ${ }^{3}$, le recueil d'Arlette et Roger Chemain ${ }^{4}$ ainsi que les travaux plus récents de Boniface MongoMboussa $^{5}$ apparaissent comme autant d'armes pour lutter contre l'oubli. Le constat est double selon ces auteurs : le Congo c'est Tchicaya, mais les Congolais l'ignorent. Un article en ligne publié à l'occasion de la parution d'une biographie du poète témoigne de ce point de vue :

« Tchicaya U Tam'si est un poète immense injustement méconnu dans son Congo natal », regrette Boniface Mongo-Mboussa. "Toute sa vie, le poète a exprimé les angoisses, les souffrances et les peurs, les paysages de tout un pays », ajoute le critique littéraire, après lecture d'un extrait de À triche-cœur, l'un des poèmes de Tchicaya U Tam'si'.

3 Les universitaires et les têtes pensantes du ministère de la culture ne sont cependant pas les seuls à vouloir entretenir la mémoire de Tchicaya, et les œuvres que nous allons 
examiner dans cet article sont même plus significatives car elles émanent d'artistes qui se réclament de cet héritage prestigieux. En effet, au début des années 1990, après la mort du poète, on dénombre au moins huit adaptations théâtrales de ses textes réalisées et mises en scène à Brazzaville ou à Pointe-Noire. Ces créations nous intéressent en ce qu'elles sont avant tout des œuvres de lecteurs souhaitant partager leur passion et s'approprier un texte même si cela implique de le découper et de le triturer. Ces différents projets témoignent également d'une transformation du théâtre pratiqué dans les deux villes principales de la République du Congo sous l'impulsion de médiateurs culturels occidentaux qui participent à la mise en scène et permettent aux productions de sortir des frontières congolaises ${ }^{7}$.

Aux problèmes posés habituellement par la génétique textuelle - à savoir la recherche de documents préparatoires et de brouillons puis l'analyse du dossier ainsi constitué s'ajoutent deux autres éléments qui complexifient singulièrement la tâche. Tout d'abord, comme il s'agit ici de réécritures, le travail de l'adaptateur s'articule autour d'un hypotexte auquel il rend hommage et qu'il cherche à mettre en valeur. À ce croisement des approches intertextuelles et génétiques, il est nécessaire d'ajouter une réflexion sur le genre, car ce sont des nouvelles, des romans et des poèmes qui sont adaptés pour le théâtre. Dans cette perspective, en plus de l'avant-texte déjà évoqué, il est nécessaire de prendre en compte ce qu'Almuth Grésillon et Nathalie Léger appellent «l'avant-scène $»^{8}$. Documents de production, notes de régie, s'ajoutent aux brouillons habituellement considérés. Justement, dans un article intitulé "Scènes de genèse théâtrale » Almuth Grésillon et Jean-Marie Thomasseau tentent de cerner les contours d'une génétique pour laquelle chaque œuvre, étant à la fois texte et représentation :

constitue de la sorte un feuilleté de substrats composites en continuel devenir, dont les interdépendances et les interactions multiples entre texte et scène, le va-etvient permanent, et la complexité des ajustements toujours renouvelés rendent l'approche génétique difficile en même temps qu'ils lancent à celle-ci un de ses plus beaux défis ${ }^{9}$.

La dynamique entre le texte et la scène.

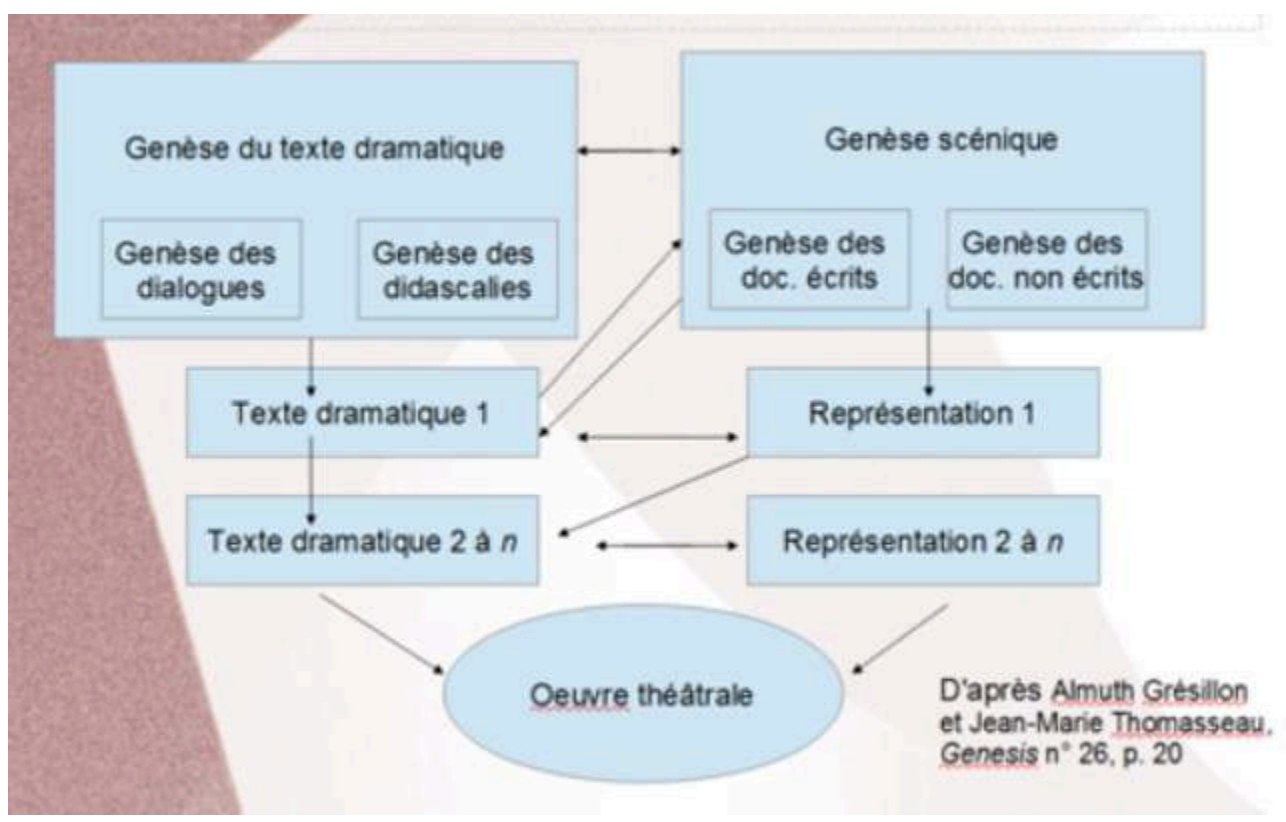

Le schéma que les deux auteurs proposent afin de rendre compte de ce phénomène montre bien la dynamique entre le texte et la scène par le biais de doubles flèches. Dans 
le cas des réécritures qui nous intéressent aujourd'hui - conformément à ce que nous venons d'exposer - nous ajoutons à cette configuration le texte-source ou hypotexte qui doit au mieux être ajouté en amont, tout en étant virtuellement présent à toutes les étapes de la création comme un point de repère et un document de travail indispensable.

Ajout de l'hypotexte au schéma précédent

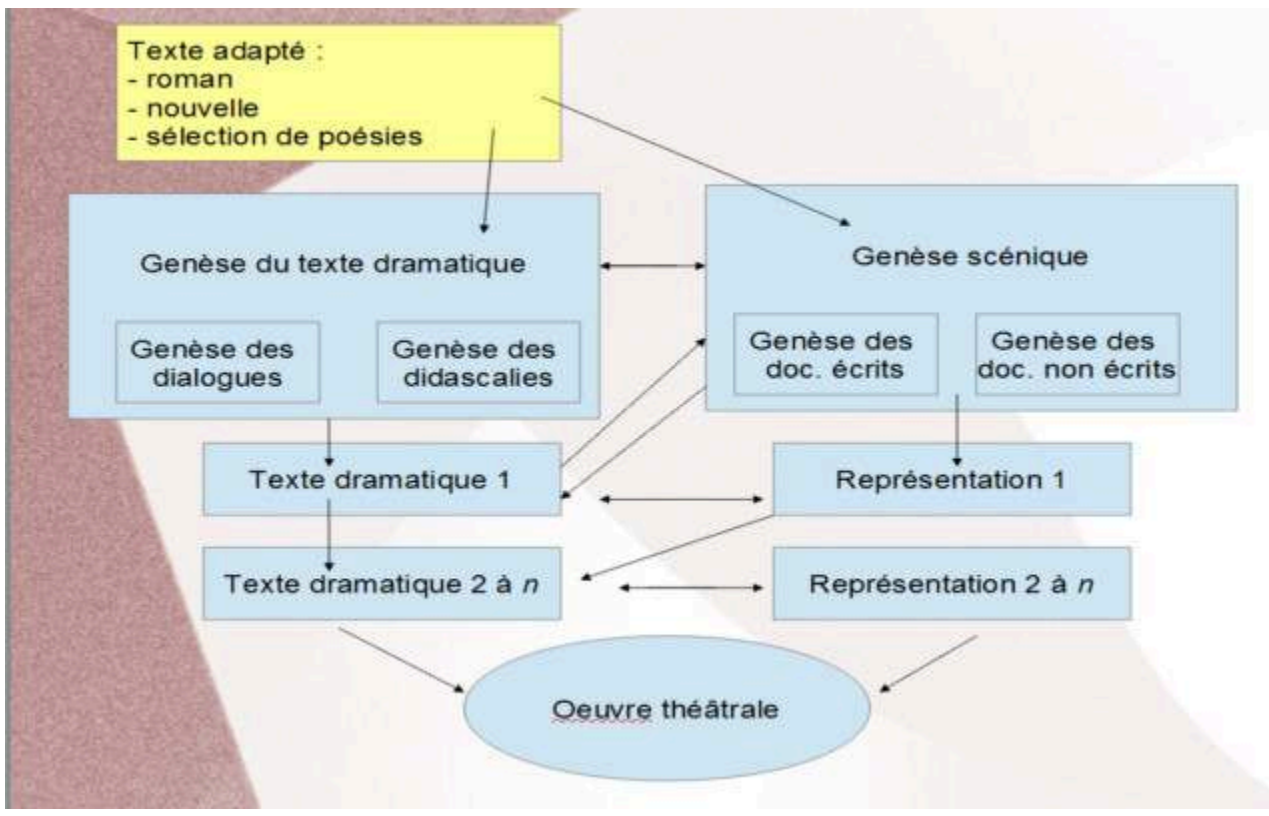

L'étude qui suit se concentrera sur trois adaptations qui présentent un caractère exemplaire du fait de la diversité des hypotextes mobilisés pour la création théâtrale. Tchicaya U Tam'si Poète congolais s'appuie en effet sur les poésies, Sékhélé sur une nouvelle et Ces fruits si doux de l'arbre à pain sur le roman éponyme. Plutôt que d'être un bilan exhaustif, cette réflexion constituera une ébauche permettant d'éprouver l'analyse de différents supports et de réfléchir à une forme particulière d'intertextualité.

\section{Tchicaya et le théâtre}

Ce n'est qu'en 1976, avec la représentation du Zulu ${ }^{10}$ à Avignon, que Tchicaya devient dramaturge. Sa seconde pièce, Le Maréchal Nnikon-Nniku, Prince qu'on sort ${ }^{11}$ (1979), écrite selon la légende lors d'un séjour en Ouganda ${ }^{12}$, semble être avant tout destinée à la lecture et ne sera montée qu'après la mort de son auteur. Enfin, le statut ambigu du dernier opus, Le Bal de Ndinga, nous semble particulièrement important car il s'agit en fait d'une nouvelle que Gabriel Garran, fondateur du Théatre international de langue française (TILF), souhaite adapter au théâtre.

La réalisation du Bal de N'Dinga, que j'ai créée à partir d'une nouvelle dialoguée a entrainé un effet "boule de neige " dès sa première création au théâtre de la Tempête, puis dans la même saison aux Bouffes du Nord, au théâtre de l'œEuvre, au Théâtre Antoine. Ce spectacle a aussi sillonné toute l'Afrique, engendrant ainsi 250 représentations. Ce fut le grand texte et le grand spectacle emblématique de la dramaturgie africaine ${ }^{13}$. 
Destiné au recueil inédit, L'Eau à contre-jour, le texte exploité par Gabriel Garran existe en deux versions sous la forme de tapuscrit et a finalement été publié dans un ouvrage collectif $^{14}$. Cette dernière "pièce» rencontre le succès alors que l'auteur vient de disparaître en avril 1988, mais elle établit en quelque sorte un précédent pour tous les spectacles que nous allons prendre en considération. Repris en 2011 par l'acteur congolais Pascal Nzonzi pour un spectacle seul en scène à la Maison de la poésie, Le Bal de Ndinga demeure aujourd'hui encore une œuvre emblématique, comme en témoigne sa diffusion sur l'antenne de France Culture le 16 octobre $2011^{15}$.

Les trois pièces de Tchicaya que nous venons très rapidement d'évoquer forment ce que Sylvie Chalaye appelle une « triade dramaturgique » balayant différents genres, et cette variété trouvera un écho dans les adaptations ultérieures :

Chaque pièce illustre en effet d'abord un genre : une tragédie shakespearienne avec Le Zulu, une comédie avec Le Bal de Ndinga et une farce avec Le Destin glorieux du maréchal Nnikon Nniku... Comme s'il s'était agi de choisir le théâtre, ou la forme dialoguée, pour éclairer un certain aspect du pouvoir, enjeu qui traverse largement son œuvre ${ }^{16}$.

\section{« Tchicaya U Tam'si poète congolais » : le procès du poète}

En 1990, le spectacle de Matondo Kubu Turé relie entre eux les poèmes de Tchicaya U Tam'si au moyen d'une trame narrative. Nous sommes ici en présence d'un cas limite pour l'étude génétique, car, selon le metteur en scène, le script a été perdu pendant la guerre civile qui a déchiré le pays entre 1997 et 2002. D'autres documents écrits ou captations n'ont pas encore été retrouvés. Nous nous appuierons donc sur un article publié dans la revue Europe $\mathrm{n}^{\circ} 750$ (octobre 1991) intitulé « Mise en scène d'une poésie, mise en verbe d'une écriture ", ainsi que sur un entretien avec Matondo Kubu Turé effectué le 9 août 2012.

L'analyse du spectacle proposée par son metteur en scène et principal acteur permet de faire le lien entre l'œuvre de Tchicaya et les adaptations ultérieures. En effet, elle suggère à la fois une qualité dramatique des poèmes, qui faciliterait le passage à la scène, et la nécessité de promouvoir une œuvre méconnue :

Dans le contexte congolais, monter des poésies de Tchicaya U Tam'si sur une scène de théâtre relève moins de la gageure que d'un combat contre une sensibilité dominante, paresseuse, qui a imposé un préjugé tenace dans le public. La scène, dans ce cas, plus qu'une autre activité, peut jouer ici le rôle paradoxal de dépasser la page écrite pour exposer le monde utam'sien comme théâtralisation d'une parole disséquée, mais toujours en affinité avec sa littérarité. Ceci tient à une chose essentielle : la veine poétique de Tchicaya $U$ Tam'si est déjà en elle-même un procès théâtral de mots, de métaphores, de gestes, de situations, de personnages jouant sur une même scène tragique ${ }^{17}$.

En considérant cette qualité dramatique du poème, Matondo Kubu Turé touche du doigt le rapport à l'oral revendiqué par Tchicaya U Tam'si ${ }^{18}$ et il souhaite récuser l'accusation selon laquelle le poète serait " ésotérique ».

Le titre même du spectacle - Tchicaya U Tam'si poète congolais - témoigne des enjeux de ce travail, et le dispositif choisi pour mettre en scène les poèmes place le poète au cœur de l'action:

La pièce construit une narration à partir de la poésie. Cette poésie raconte le drame du Congo du début jusqu'à la fin. L'interlude représente une sorte de tribunal qui accuse Tchicaya d'être ésotérique. À cette occasion on lui pose ces questions : «Mais pourquoi vous écrivez comme ça? Pourquoi vous ne voulez pas vous faire 
entendre des gens? » Dans sa poésie, il y a un certain nombre de parties dans lesquelles il répond à ces questions-là. Le spectacle commence donc par ce tribunal et la suite illustre ces propos parce qu'il a dit: «mon écriture n'est pas difficile, c'est le Congo qui est difficile. C'est le Congo que vous n'arrivez pas à comprendre ${ }^{19}$. »

En proposant un procès, Matondo Kubu Turé donne un contexte aux différents poèmes et il construit par la même occasion une défense de l'auteur par ses propres textes. Ainsi, les quelques éléments à notre disposition nous donnent l'image d'un spectacle qui se préoccupe autant d'une figure littéraire que d'un ensemble de poèmes. Le poète, Tchicaya U Tam'si, finit par incarner « le drame du Congo ».

\section{Pointe-Noire : « Le Fou rire »}

11 La configuration est différente dans le cas du spectacle Sékhélé, adapté de la nouvelle «Le Fou rire », elle-même tirée du recueil La Main sèche. En effet, après une première version réalisée à Pointe-Noire par un cousin de Tchicaya U Tam'si, Tchicaya JeanRobert (alias Tchicaya Unti Bukune, « la petite plante qui plante »), le metteur en scène suisse Jean-Pierre Amiel, s'intéresse au projet et commande une nouvelle adaptation aux universitaires Arlette et Roger Chemain. La troupe Punta Negra se monte alors autour d'un projet d'atelier d'art dramatique dirigé par Amiel, et la pièce, contrairement au spectacle de Matondo Kubu Turé, connaît une série de représentations en Europe et en Amérique du Nord de 1991 (première représentation en Suisse) à 1995 (mai 1995 à Victoria, Canada). Le dossier génétique, encore maigre, est tout de même plus conséquent que dans le premier cas étudié. Nous disposons d'un scénario sans annotations, d'un extrait publié dans $L a$ Revue Noire ${ }^{20}$ ainsi que d'extraits filmés.

12 Parmi tous les textes de La Main sèche, "Le Fou rire », parce qu'il situe l'action au cœur du marché populaire et par l'importance accordée au corps de son héros, contient déjà un certain nombre de caractéristiques de la farce qui facilitent son adaptation pour la scène. Le personnage principal, un mendiant, fait rire le public par ses pets. Ce rire est gratuit pour le peuple mais payant pour les puissants. Ceux qui refusent de s'acquitter de leur dette meurent dans les plus brefs délais. Chaque acte de l'adaptation s'organise autour de l'un de ces épisodes. C'est tout d'abord le prévôt des marchands surnommé " panse de buffle, foie de requin » qui fait les frais du rire dévastateur. Vient ensuite le tour d'un éleveur de crocodiles et c'est enfin le président lui-même qui succombera après avoir défié le mendiant Sékhélée ${ }^{1}$.

13 La question de la coïncidence entre personnage et acteur évoquée en introduction prend dans ce spectacle un sens particulier car la figure du conteur - équivalent scénique du narrateur dans la nouvelle - se confond avec celle du mendiant. Ainsi, Sékhélé lui-même prononce la dernière réplique et conclut son histoire en employant la troisième personne du singulier :

On retrouva le corps de Sékhélé l'œil sec, il était vide, sa peau était vide à l'intérieur ; pas un os, pas un bout de chair, qui était cet homme?

On vint encore dire au juge qu'on avait vu Sékhélé à 4 endroits de la ville. On l'a vu à Poto-Poto, à Ouenzé, à Bacongo, à Talangaï et à... (nom de la ville où le spectacle se joue $)^{22}$ 
Ce dispositif dans lequel le personnage raconte sa propre absence illustre bien le paradoxe incarné par Sékhélé. Le corps rempli d'air qui fait rire par ses pets n'est plus qu'une enveloppe vide à la fois partout et nulle part.

\section{Ces fruits si doux de l'arbre à pain}

14 Ce spectacle monté par Antoine Yirrika en 1994 avec l'aide du Centre Culturel Français ( $\mathrm{CCF}$ ) de Brazzaville et la collaboration de Christian Remer à la mise en scène, présente un caractère plus ambitieux que le précédent dans le sens où il s'agit de l'adaptation d'un roman. Cette fois, les documents à notre disposition sont plus variés et plus nombreux. Nous disposons de deux versions du texte - l'une annotée en vue du spectacle et l'autre "au propre ", destinée à la publication - ainsi que d'un montage vidéo promotionnel qui nous permet de visualiser la scénographie et le jeu des acteurs. L'entretien avec Antoine Yirrika qui a bien voulu partager avec nous ces documents a d'ailleurs eu lieu dans les locaux de l'association « Tchicaya U Tam'si » qu'il a fondée à Brazzaville.

Le roman Ces fruits si doux de l'arbre à pain raconte l'histoire du juge Raymond Poaty, un haut magistrat congolais qui, quelques années après l'indépendance, s'interroge sur une série de meurtres qui trouverait son origine au sommet de l'État. Constatant qu'il ne peut rien faire en suivant la voie légale, il prend position publiquement, et, alors qu'on le surnomme "le prophète Raymond ${ }^{23}$, il disparait mystérieusement, sans doute pour être torturé et tué en secret. À la suite de ce rapt, c'est le fils de Raymond, Gaston, qui passe sur le devant de la scène. Une révolution a lieu, le chef corrompu tombe et un régime marxiste se met en place. L'histoire se répétera pourtant et Gaston devra s'enfuir pour échapper à la mort. En parallèle de cette trame urbaine, le lecteur prend connaissance d'une suite de contes racontés par un personnage mystérieux, la jeune Mouissou. Les récits proférés au village, autour du feu de la veillée, redoublent et commentent le parcours des Poaty. La complexité de l'intrigue que nous ne faisons ici qu'effleurer constitue un défi pour toute tentative de transposition qui doit trouver un équilibre entre une simplification excessive et un foisonnement inintelligible.

Dans son travail d'adaptation, Antoine Yirrika a privilégié la fidélité et le collage d'extraits tout en jouant sur la distribution de la parole pour faire du récit un discours. Afin de rendre compte du travail de sélection et de réduction, il suffit de prendre un paragraphe situé à la fin du second chapitre et d'observer ce qu'il devient dans l'adaptation. Dans le roman, donc, une longue description de paysage permet de mettre en valeur le " tchilolo", arbre totémique de la famille Poaty :

À perte de vue, quand, de leurs flammes gloutonnes, les feux de brousse ont brûlé à ras l'herbe de la savane et n'ont laissé après cette infernale fenaison que des éteules noires, on voit, ça et là, un arbuste à feuilles persistantes, aux racines traçantes, qui saigne rouge, que l'on nomme: tchilolo. Sa taille n'est pas plus haute que celle d'un homme accroupi. À peine un enfant - pas un homme en tout cas - se cacherait derrière son feuillage, peu abondant d'ailleurs. Ses fruits en grappe sont des papayes en miniature, d'où le nom qu'on leur donne : bilolo. Comme les fruits du papayer, ils sont du même vert sombre quand ils ne sont pas mûrs, jaune quand ils le sont. Cependant, ni à l'aspect ni au goût on ne peut assimiler les uns aux autres. À l'intérieur, le fruit du tchilolo se fragmente comme une mandarine. Sa [La] chair [de son fruit] est sanglante comme sa racine. Elle n'est pas juteuse, au contraire, mais très ferme et surtout farineuse. En fait, il y a un fruit qui a ce côté farineux et qui lui est 
comparable : [S'il y a comparaison à faire, ce fruit est comparable à] celui de l'arbre à pain, autrement dit le fruit à pain ${ }^{24}$.

On constate aisément que, tout en restant fidèle, le compilateur réduit le texte de manière drastique et seules demeurent les quelques lignes ci-dessous :

Arbuste à feuilles persistantes, aux racines traçantes, qui saigne rouge. Sa taille est celle d'un homme accroupi. Ses fruits en grappe sont des papayes en miniature, Le fruit et l'arbuste ont le même nom. La chair de son fruit est sanglante comme sa racine. Pas juteuse mais ferme et surtout farineuse. S'il y a comparaison à faire, ce fruit est comparable à celui de l'arbre à pain ${ }^{25}$.

Il y a là très peu de transformations et les quelques formules qui ne relèvent pas directement du collage servent à résumer le texte romanesque et à lier entre elles les propositions sélectionnées.

17 Comme pour le conteur dans Sékhélé, se pose le problème de l'énonciation et de l'exposition. Qui doit prendre en charge la narration tchicayenne? Encore une fois, l'examen de ce prologue nous renseigne sur un processus qui se prolonge au-delà de l'écriture d'une première version. Le texte dactylographié indique un seul acteur pour l'ensemble du prologue mais les ajouts au stylo bleu proposent une répartition de la parole qui ne se fait pas en fonction de personnages mais de personnes, puisque ce sont les acteurs, Nicolas Bissi, Flore MBongo, Louis Mouboundou et Alphonsine Moundélé, qui sont désignés. L'influence réciproque entre le texte écrit et les nécessités de la scène telle qu'elle est décrite par Almuth Grésillon est bien présente ici. Dans ce cas de figure, le texte est bien avant tout un support pour la préparation du spectacle. La présentation neutre dans le document mis au propre marque, elle, un détachement du texte par rapport à cette mise en scène particulière. Le terme acteur revient d'ailleurs pour désigner les locuteurs.

Prologue de l'adaptation de Ces fruits si doux de l'arbre à pain par Antoine Yirrika, version de travail annotée.

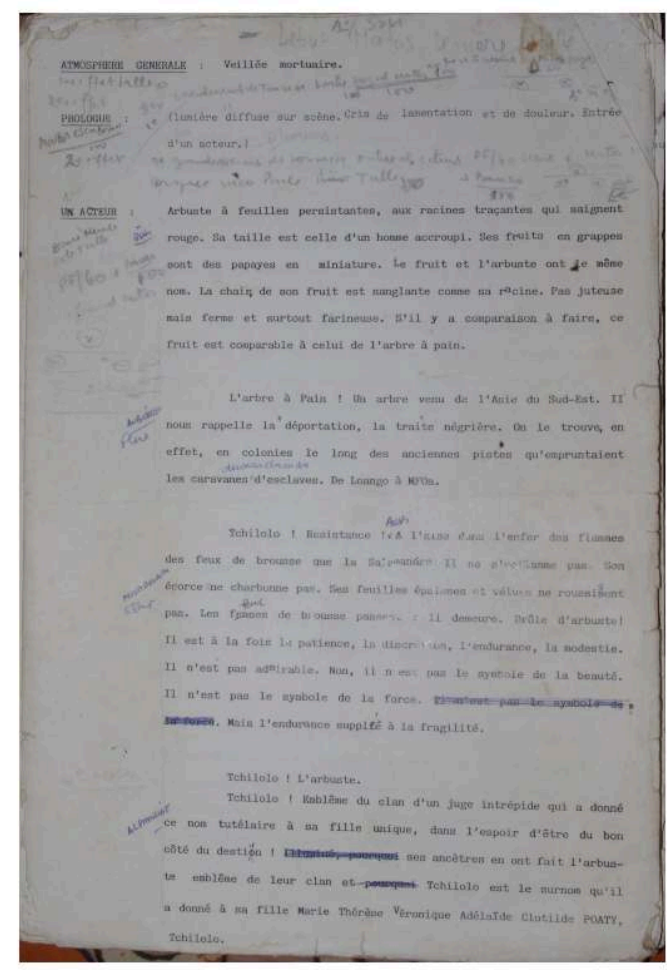


Prologue de l'adaptation de Ces fruits si doux de l'arbre à pain par Antoine Yirrika, version « au propre " destinée à la publication.

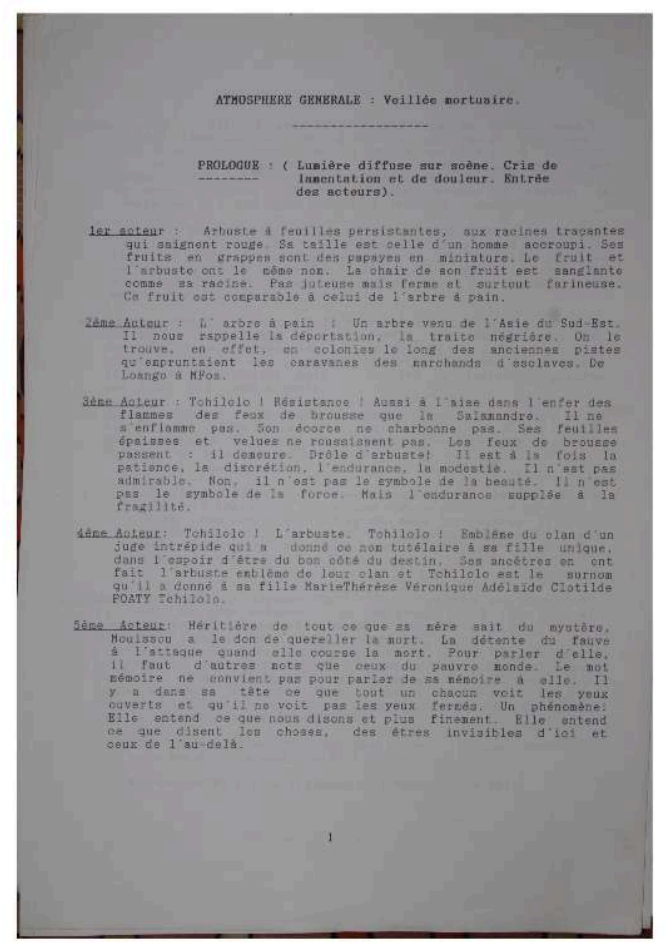

Le genre théâtral impose le dialogue là où dominait la narration et, dans certains cas, ce passage d'un genre à l'autre peut figer l'interprétation en mettant par exemple dans la bouche d'Isidore, ami et admirateur du juge Poaty, des paroles presque inassignables dans le roman. Ainsi, le texte de Tchicaya évoque des justifications apportées au comportement général du juge et celles-ci peuvent aussi bien émaner d'un flatteur que du personnage lui-même :

La porte a toujours été ouverte à ses désirs, pour ne pas dire ses ambitions. L'optimisme mouille de ses eaux bienfaisantes les eaux de son cœur. Il se gagne ainsi des sympathies qui sont nécessaires à sa cause. Sa cause est le sanctuaire d'un grand dessein. Il est juste qu'il soit juste, puisque, par la grâce de Dieu, il est juge des actions des hommes ${ }^{26}$.

La version adaptée par Antoine Yirrika, en assignant cette parole à un personnage, réduit les possibilités d'interprétation et le passage à la deuxième personne du singulier nous rappelle qu'il s'agit là d'une conversation:

La porte a toujours été ouverte à tes désirs, pour ne pas dire tes ambitions. L'optimisme mouille de ses eaux bienfaisantes les eaux de ton cœur. Tu te gagnes ainsi des sympathies qui sont nécessaires à ta cause. Ta cause est le sanctuaire d'un grand dessein. Il est juste que tu sois juste, puisque, par la grâce de Dieu, tu es juge des actions des hommes ${ }^{27}$.

19 Le but de cette démarche n'est pas pour autant de figer le texte en explicitant des passages jugés obscurs. La mise en scène, que l'on peut reconstituer à partir de nombreuses notes sur le document de travail, crée de nouvelles ambiguïtés en s'appuyant sur un langage qui lui est propre. La coïncidence acteur-personnage joue comme pour le conteur-Sékhélé - un rôle déterminant dans la transformation du roman en spectacle. Les indications sur le brouillon, ainsi que les courts extraits de la 
captation, nous permettent de voir que le juge Raymon Poaty et son fils Gaston sont incarnés par un seul acteur: Matondo Kubu Turé. Ce choix scénique rétablit en quelque sorte l'ambivalence originelle du roman. Après avoir distribué une voix composite entre plusieurs acteurs, un seul acteur assume deux personnages qui se succèdent dans le roman.

\section{Conclusion} dossiers génétiques de ces adaptations et les difficultés déjà grandes en ce qui concerne les textes édités au Congo sont encore renforcées pour ces productions par essence éphémères. Les trois exemples choisis pour cet article sont les plus significatifs mais ils ne sont pas uniques et on retrouve d'autres projets sur le sol congolais ${ }^{28}$ ou à l'étranger ${ }^{29}$. Le phénomène, à Brazzaville et à Pointe-Noire, témoigne de l'aura d'un auteur qui a pourtant quitté son pays natal à l'âge de 15 ans pour y revenir de manière sporadique. Sa célèbre assertion concernant un de ses collègues de la "phratrie » des écrivains congolais - «Je dirais que Sony habite le Congo, moi le Congo m'habite ${ }^{30}$.» est assurément plus vraie encore depuis que des lecteurs - pour la plupart congolais se sont emparés de ses textes. traitement qui semble être à la fois un moyen de canoniser des écrivains et d'enrichir le patrimoine théâtral. Dès 1981, La troupe Ngunga, à laquelle appartient Matondo Kubu Turé, monte Les Bouts de bois de Dieu de Sembène Ousmane. Antoine Yirrika, en plus de Ces fruits si doux de l'arbre à pain, a aussi adapté plusieurs textes et ensembles poétiques de Jean-Baptiste Tati-Loutard. Matondo Kubu Turé a créé - après Tchicaya U Tam'si Poète congolais - un spectacle sur Aimé Césaire. Enfin, Christian Remer, qui a collaboré à la mise en scène de Ces fruits si doux de l'arbre à pain, était venu au Congo pour monter un autre adaptation : le recueil de nouvelles Jazz et Vin de Palme d'Emmanuel Dongala.

De la mise en procès du poète à la création d'un personnage double, les différentes réécritures de Tchicaya U Tam'si - qu'elles tirent leur substance de la poésie, d'une nouvelle ou d'un roman - s'affirment avant tout comme des réflexions sur le lieu de l'énonciation et la distribution de la parole. Les lecteurs, qui dans certains cas sont aussi acteurs, souhaitent reprendre les mots du poète afin de prolonger son œuvre et de dépasser les réserves du public à son égard. L'exercice n'est ni unique ni nouveau et il est intéressant, pour les trois ensembles étudiés, de reprendre les mots de François Regnault qui avait inventé un prologue à La Dispute de Marivaux en combinant divers extraits des textes de l'auteur: "Tout le grain est authentique, le tamis seul est d'emprunt $^{31}$." Ces œuvres nouvelles qui naissent des textes de Tchicaya et qui s'inscrivent dans une même période historique témoignent de la place particulière du poète dans son pays d'origine et de son influence sur le monde de la culture. 


\section{BIBLIOGRAPHY}

Agence d'information d'Afrique centrale, 26 juillet 2014, « Tchicaya U Tam'si : un poète injustement méconnu, défendu par Boniface Mongo-Mboussa », en ligne : http://www.adiaccongo.com/content/tchicaya-u-tamsi-un-poete-injustement-meconnu-defendu-par-bonifacemongo-mboussa-17493 (consulté le 5 septembre 2014).

Chalaye, Sylvie, « Les Trois Coups de théâtre de Tchicaya U Tam'si, Cultures Sud n 171, octobredécembre 2008.

Chemain-Degrange, Arlette, Chemain, Roger, De Gérald Félix-Tchicaya à Tchicaya U Tam'si : hommage, Paris, L'Harmattan, 2008.

Chiappano, Nino, Tchicaya notre ami : l'homme, l'œuvre, l'héritage, Paris, A.A.F.U : Agence de la francophonie, 1998.

Garran, Gabriel, « L’Empreinte de Tchicaya », Cultures Sud n 171, octobre-décembre 2008.

Grésillon, Almuth, Léger, Nathalie, « Brouillons de l'éphémère, pour une génétique du théâtre », Genesis $n^{\circ} 25,2005$.

Grésillon, Almuth, Thomasseau, Jean-Marie, « Scènes de genèse théâtrale ", Genesis n 25, 2005.

Kubu Turé, Matondo, « Mise en scène d'une poésie, mise en verbe d'une écriture », Europe $\mathrm{n}^{\circ} 750$, octobre 1991.

Ngalibo-Yala, Pascal, « Vingt-huit bustes ont été dévoilés, rue Melvin Jones, à Brazzaville », La Semaine africaine du 14 août 2012.

Mongo-Mboussa, Boniface, Tchicaya U Tam'si, Le viol de la lune : Vie et œuvre d'un maudit, La Roqued'Anthéron, Vents d'ailleurs, 2014.

«Sékhélé, Tchicaya U Tam'si, Compagnie Punta Negra », La Revue Noire ${ }^{\circ}$ 10, septembre-octobre 1993.

Marivaux, Les Acteurs de bonne foi, La Dispute, L'Épreuve, édition de Jean Goldzink, G.F., Paris, Flammarion, 1991.

Montrémy, Jean-Maurice de, « Tchicaya U Tam'si, écrivain de l'Afrique et du destin », La Croix, 12-13 octobre 1980

Naumann, Michel, «Culture orale et contemporaine et écriture d'une nouvelle de Tchiaya U Tam'si », Mots Pluriels n 9, février 1999.

Tchicaya U Tam'si, Ces fruits si doux de l'arbre à pain, Paris, Seghers, 1987.

Tchicaya U Tam'si, La Main sèche, Paris,Robert Laffont, 1980.

Tchicaya U Tam'si, Le Destin glorieux du maréchal Nnikon-Nniku, Prince qu'on sort, Paris, Présence Africaine, 1979.

Tchicaya U Tam'si, Le Zulu, suivi de Vuene le fondateur, Paris, Nubia, 1977. 


\section{NOTES}

1. Cet ensemble placé près de la gare du CFCO (chemin de fer Congo-Océan) entre dans le projet de monuments historiques prévu par le gouvernement congolais. Le discours du directeur général du Patrimoine et des Archives, Samuel Kidiba, est à ce sujet éclairant : « Ils ont, ainsi, par leur action, leur talent et leur envergure, dans les disciplines, leurs activités et leurs ordres, participé à l'élaboration et à l'éclosion des créativités littéraires, spirituelles, médiatiques et sportives qui ont façonné les imaginaires de l'homme congolais moderne.» (cité par Pascal Ngalibo-Yala in "Vingt-huit bustes ont été dévoilés, rue Melvin Jones, à Brazzaville », La Semaine africaine du 14 août 2012).

2. La plupart des documents présentés ici ont été rassemblés lors d'un travail de terrain effectué à Brazzaville et Pointe-Noire en août 2012. Cet article reprend et adapte le texte d'une séance du séminaire de l'ITEM du 18 janvier 2013.

3. Chiappano, Nino, Tchicaya notre ami: l'homme, l'oeuvre, l'héritage, A.A.F.U: Agence de la francophonie, 1998.

4. Chemain-Degrange, Arlette, Chemain, Roger, De Gérald Félix-Tchicaya à Tchicaya U Tam'si: hommage, Paris, L'Harmattan, 2008.

5. Mongo-Mboussa, Boniface, Tchicaya U Tam'si, Le viol de la lune: Vie et œuvre d'un maudit, La Roque-d'Anthéron, 2014.

6. Agence d'information d'Afrique centrale, 26 juillet 2014, "Tchicaya U Tam'si: un poète injustement méconnu, défendu par Boniface Mongo-Mboussa », en ligne: http://www.adiaccongo.com/content/tchicaya-u-tamsi-un-poete-injustement-meconnu-defendu-par-bonifacemongo-mboussa-17493 (consulté le 5 septembre 2014).

7. Nous pouvons citer ici le metteur en scène Christian Remer pour Ces fruits si doux de l'arbre à pain ou les universitaires Arlette et Roger Chemain pour l'adaptation de la nouvelle « Le Fou rire ». Dans ce dernier cas, notons que le critique se fait auteur et transformateur du texte qu'il commente.

8. Grésillon, Almuth, Léger, Nathalie, «Brouillons de l'éphémère, pour une génétique du théâtre ", in Genesis n ${ }^{\circ}$ 25, 2005, p. 7.

9. Grésillon, Almuth, Thomasseau, Jean-Marie, "Scènes de genèse théâtrale ", in Genesis $\mathrm{n}^{\circ}$ 25, 2005, p. 19.

10. Tchicaya U Tam'si, Le Zulu, suivi de Vuene le fondateur, Paris, Nubia, 1977.

11. Tchicaya U Tam'si, Le Destin glorieux du maréchal Nnikon-Nniku, Prince qu'on sort, Paris, Présence Africaine, 1979.

12. Mukala Kadima-Nzuji, entretien effectué le 3 août 2012.

13. Garran, Gabriel, «L'empreinte de Tchicaya », Cultures Sud $n^{\circ} 171$, octobre-décembre 2008, p. 112.

14. L'Atelier imaginaire, Paris, éd. l'Âge d'Homme, 1987, p. 169-185.

15. La première diffusion a eu lieu le 16 octobre 2011 à $22 \mathrm{~h}$ et l'émission est restée un an en écoute sur Internet. Une fiche concernant ce programme est disponible à l'adresse suivante : http://www.franceculture.fr/emission-fictions-theatre-et-cie-11-12-le-bal-de-ndinga-2011-10-16 (consulté le 6 novembre 2014).

16. Chalaye, Sylvie, «Les trois coups de théâtre de Tchicaya U Tam'si, Cultures Sud ${ }^{\circ} 171$, octobre-décembre 2008, p. 71.

17. Kubu Turé, Matondo, "Mise en scène d'une poésie, mise en verbe d'une écriture ", in Europe $\mathrm{n}^{\circ} 750$, octobre 1991 p. 127-128.

18. Dans un entretien, Tchicaya expose ainsi une méthode de travail qui associe la voix au travail d'écriture : «Paradoxalement, je n'aime pas les choses écrites. Je travaille... au magnétophone ! Comme dirait Flau, je "gueule". Enfin, pas systématiquement. Puisque c'est destiné à être imprimé, je dois bien m'astreindre à tracer des signes. Mais j'aime surtout palabrer, discuter. » 
( Tchicaya U Tam'si, écrivain de l'Afrique et du destin » Entretien avec J.-M. de Montrémy pour le quotidien La Croix, 12-13 octobre 1980).

19. Entretien avec Matondo Kubu Turé le 9 août 2012.

20. «Sékhélé, Tchicaya U Tam'si, Compagnie Punta Negra », in La Revue Noire n 10, septembreoctobre 1993, p. 6-7.

21. Pour une analyse plus détaillée de cette nouvelle et une réflexion sur l'oralité dans l'œuvre de Tchicaya U Tam'si, on consultera avec profit l'article de Michel Naumann, «Culture orale et contemporaine et écriture d'une nouvelle de Tchicaya U Tam'si » in Mots Pluriels $\mathrm{n}^{\circ} 9$, février 1999.

22. Sékhélé, tapuscrit inédit non daté, p. 25.

23. Tchicaya U Tam'si, Ces fruits si doux de l'arbre à pain, Paris, Seghers, 1987, p. 143.

24. Ibid., p. 17-18. Nous soulignons les passages repris tels quels dans l'adaptation.

25. Yirrika, Antoine, Ces fruits si doux de l'arbre à pain, tapuscrit inédit non daté, version de travail annotée, p. 1. Nous soulignons les ajouts.

26. Tchicaya U Tam'si, Ces fruits si doux de l'arbre à pain, op.cit., p. 38.

27. Yirrika, Antoine, Ces fruits si doux de l'arbre à pain, op.cit., p. 7. Nous soulignons.

28. En août 2012, au moment de notre enquête à Brazzaville, la troupe du théâtre national préparait une adaptation de «Nkollé » un conte tiré de l'anthologie Légendes Africaines compilée par Tchicaya.

29. Christian Remer a mis en scène au Havre, toujours avec la compagnie Punta Negra, le spectacle Un vaurien chez les sauriens adapté de la nouvelle "Noces» que l'on trouve dans le recueil La Main sèche.

30. Cité dans Chiappano, Nino (éd.), Tchicaya notre ami, l'homme, l'œuvre, l'héritage, Paris, Agence de la francophonie, 1998.

31. Cité par Jean Goldzink in Marivaux, Les Acteurs de bonne foi, La Dispute, L'Épreuve, édition de Jean Goldzink, Paris, G.F. Flammarion, 1991, p. 104.

\section{ABSTRACTS}

As a playwright, the congolese writer Tchicaya U Tam'si (1931-1988) has only authored three plays : Le Zulu, Le Destin glorieux du Maréchal Nnikon-Nniku prince qu'on sort and Le Bal de Ndinga. Nonetheless, many theatrical adaptations of his novels, short stories and poems reveal both Congolese and French artists wished to re-appropriate the legacy left by U Tam'si they used to call "the father of our dream". The analysis of three performances based upon U Tam'si's writings and put on in Brazzaville and Pointe-Noire shows how the stage transforms the texts and how the real author becomes a fiction character.

L'œuvre théâtrale de l'auteur congolais Tchicaya U Tam'si (1931-1988) se résume à trois textes : Le Zulu, Le Destin glorieux du Maréchal Nnikon-Nniku prince qu'on sort et Le Bal de Ndinga. Cependant, les nombreuses adaptations pour la scène de romans, nouvelles et autres ensembles de poésies témoignent de la volonté qu'ont eue certains artistes congolais ou français de se réapproprier l'héritage du "père de notre rêve ". Au travers de l'examen de trois spectacles montés à Brazzaville et à Pointe-Noire, cet article tente de montrer comment la scène transforme les textes et comment l'auteur lui-même devient un personnage représenté et fantasmé. 
AUTHOR

PIERRE LEROUX

Doctorant, université Paris III 